\title{
Magnetic Behaviour of Ferritin Nanoparticles
}

\author{
M. MigLIERINI ${ }^{a, *}$ AND A. LANCOK ${ }^{b}$ \\ ${ }^{a}$ Slovak University of Technology, Ilkovičova 3, 81219 Bratislava, Slovakia \\ ${ }^{b}$ Institute of Inorganic Chemistry AS CR, v. v. i., 25068 Husinec-Řež 1001, Czech Republic
}

\begin{abstract}
Characterization of magnetic behaviour of ferritin nanoparticles of biological origin is presented. Two types of samples prepared from human and horse spleen tissues were investigated by ${ }^{57} \mathrm{Fe}$ Mössbauer spectrometry. At room temperature, ferritin nanoparticles exhibit superparamagnetic behaviour due to their small dimensions. First occurrence of magnetic interactions was noticed below $60 \mathrm{~K}$. Blocking temperature of human and horse ferritin nanoparticles was determined to be of $16 \mathrm{~K}$ and $32 \mathrm{~K}$, respectively.
\end{abstract}

PACS numbers: 76.80.+y, 31.30.Gs, 75.75.-c

\section{Introduction}

Ferritin is one of basic iron-storage proteins present in the cytoplasma of cells and, to lower extent, also in blood circulation [1]. It is one of the major proteins of iron metabolism. Ferritin creates spherical formations with the size of $12 \mathrm{~nm}$. The core of ferritin is $8 \mathrm{~nm}$ large and consists of ferrihydrite $-5 \mathrm{Fe}_{2} \mathrm{O}_{3} \cdot 9 \mathrm{H}_{2} \mathrm{O}[2]$. In excessive volume of iron in the organism, Fe is stored in cells in the form of hemosiderin [3]. Hemosiderin is considered to be a proteolytic product of ferritin [4].

\section{Experimental}

${ }^{57} \mathrm{Fe}$ Mössbauer spectrometry is a useful method of characterization of iron structural positions in biological samples $[5,6]$. In addition, information on magnetic states of $\mathrm{Fe}$ atoms is readily available. This contribution aims in characterization of magnetic states of ferritin nanoparticles contained in human and horse spleen tissue. Ferritin samples have been prepared by common methods [7].

${ }^{57} \mathrm{Fe}$ Mössbauer spectrometry was carried out using a standard constant acceleration spectrometer using a ${ }^{57} \mathrm{Co}$ source embedded in a rhodium matrix. Transmission geometry experiments were performed in temperature range 5-300 $\mathrm{K}$ using liquid helium bath cryostat (SVT-400 Variable Temperature Cryostat Janis).

\section{Results and discussion}

Selected examples of the Mössbauer spectra collected from human and horse ferritin at different temperatures are illustrated in Fig. 1. At room temperature $(300 \mathrm{~K})$, two-line absorption spectra are observed that are characteristic for non-magnetic material. In fact, ferritin nanoparticles exhibit superparamagnetic behaviour due

* corresponding author; e-mail: marcel.miglierini@stuba.sk to dynamic spin relaxation process of their magnetic moments at high enough temperature. These spectra were fitted with two doublets, one of them with isomer shift and quadrupole splitting $(Q S)$ parameters indicative of the presence of the ferrihydrite core of hemosiderin and ferritin [6]. Its relative contribution is of $84 \%$ and $75 \%$ in human and horse ferritin, respectively. Spectral parameters of the second doublet are notably distinct in both types of ferritin. Consequently, an apparent asymmetry of the Mössbauer spectrum taken at $300 \mathrm{~K}$ is observed in Fig. 1a in comparison with that in Fig. 1b.

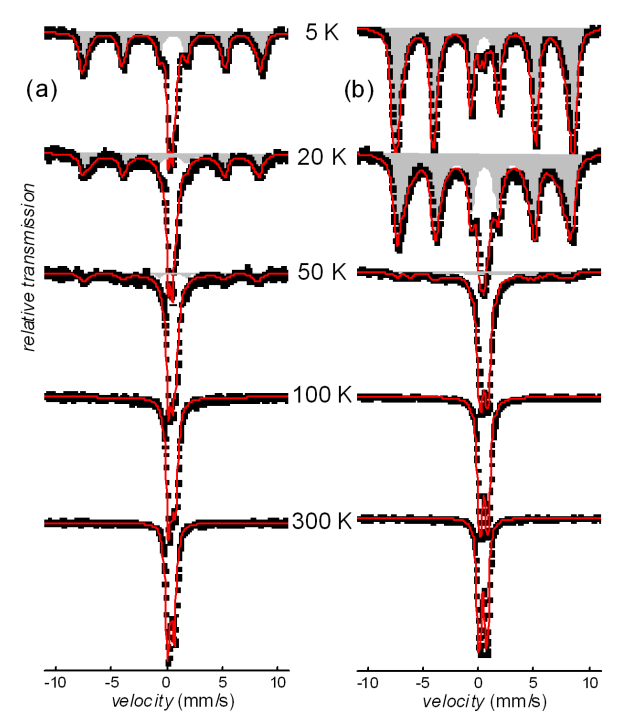

Fig. 1. ${ }^{57}$ Fe Mössbauer spectra of human (a) and horse (b) ferritin taken at the indicated temperatures. Magnetic components are coloured in gray.

As the temperature of measurement decreases, the magnitude of the magnetic hyperfine field gradually increases due to reduced precession of the particle's magnetization vector. Subsequently, six-line absorption spectra characterized by broadened sextets appear at about $50 \mathrm{~K}$. They are attributed to magnetically ordered nanopar- 
ticles and their corresponding spectral components are marked by grey colour in Fig. 1. It is noteworthy that their contribution in the horse ferritin is more pronounced.

Quantitative analysis of the obtained Mössbauer spectra parameters is illustrated by the help of Figs. 2 and 3. Temperature dependences of weighted averages $\langle Q S\rangle$ obtained from quadrupole splittings of both doublets present in the Mössbauer spectra of human and horse ferritin are plotted in Fig. 2. Structural differences are demonstrated by significant distinctions in $\langle Q S\rangle$ at room temperature. This dissimilarity in $\langle Q S\rangle$ is practically unchanged down to about $50 \mathrm{~K}$ when it starts to vanish. At $5 \mathrm{~K}$, both types of ferritin show almost equal $\langle Q S\rangle$ values though the relative content of paramagnetic regions is quite different as discussed below.

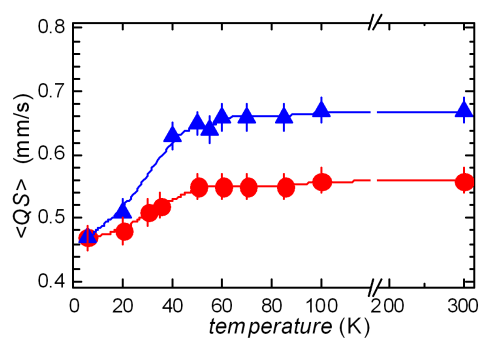

Fig. 2. Average quadrupole splitting $\langle Q S\rangle$ plotted against temperature as obtained from the Mössbauer spectra of human (circles) and horse (triangles) ferritin.
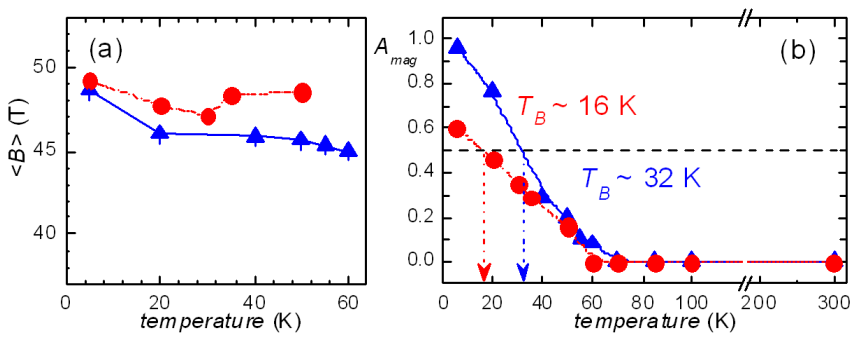

Fig. 3. Average hyperfine magnetic field $\langle B\rangle$ (a) and relative area $A_{\text {mag }}$ (b) of magnetic components in the Mössbauer spectra of human (circles) and horse (triangles) ferritin plotted against temperature. Blocking temperatures $T_{\mathrm{B}}$ are indicated by arrows.

Traces of magnetically ordered ferritin are revealed in the Mössbauer spectra (Fig. 1) by six-line spectral components. Average magnetic hyperfine fields $\langle B\rangle$ and relative fraction of this magnetic part $A_{\text {mag }}$ are plotted against temperature in Fig. 3a and Fig. 3b, respectively. The onset of magnetic phase is determined to be at $50 \mathrm{~K}$ and $60 \mathrm{~K}$ for human and horse ferritin, respectively.
The so-called blocking temperature, $T_{\mathrm{B}}$, of the ensemble of ferritin nanoparticles in the investigated samples is the temperature at which the shape of the Mössbauer spectrum changes from magnetic sextuplet into paramagnetic doublet. Here, we have estimated $T_{\mathrm{B}}$ as a temperature at which the absorption area of the Mössbauer spectrum consists of $50 \%$ magnetic and $50 \%$ paramagnetic components. From Fig. $3 \mathrm{~b}, T_{\mathrm{B}}$ of human and horse ferritin is determined to be of about $16 \mathrm{~K}$ and $32 \mathrm{~K}$, respectively. It is noteworthy that $T_{\mathrm{B}}$ is not an intrinsic property of the nanoparticles but rather a technique dependent parameter that records spin dynamical behaviour relative to the time window of the experimental measurement [8].

\section{Conclusions}

In this work, we have investigated magnetic properties of ferritin nanoparticles of biological origin which were derived from human and horse spleen tissues. The principal constituent of ferritin was shown to be ferrihydrite. Depending on temperature, its superparamagnetic behaviour changes and below the blocking temperature more than $50 \%$ of agglomerates of ferritin nanoparticles exhibit magnetic states.

\section{Acknowledgments}

This work was supported by the grants VEGA 1/0033/10, GACR P204/10/0035, and AV0Z40320502. We are grateful to J. Kohout (Prague) for assistance with low temperature Mössbauer measurements. Ferritin samples were provided by courtesy of M. Kopani (Bratislava).

\section{References}

[1] P. Arosio, S. Levi, Free Radical Biol. Med. 33, 457 (2002).

[2] N.D. Chasteen, P.M. Harrison, J. Struct. Biol. 126 , 182 (1999).

[3] C. Seldon, M. Owen, J.M. Hopkins, T.J. Peters, Br. J. Haematol. 44, 593 (1980).

[4] M.P. Weir, J. F Gibson, T. J Peters, Biochem. J. 223, 31 (1984).

[5] M.I. Oshtrakh, J. Radioanal. Nucl. Chem. 269, 407 (2006).

[6] D. Meyrick, J. Webb, C. Cole, Inorg. Chim. Acta 339, 481 (2002).

[7] M. Kopani, J. Jakubovsky, S. Polak, Acta Phys. Slov. 51, 339 (2001).

[8] G.C. Papaefthymiou, Nano Today 4, 438 (2009). 